\title{
EQUIVARIANT SINGULAR HOMOLOGY AND COHOMOLOGY
}

\author{
BY SÖREN ILLMAN \\ Communicated by W. Browder, July 3, 1972
}

Let $G$ be a topological group. By a $G$-space $X$ we mean a topological space $X$ together with a left action of $G$ on $X$. Under the assumption that $G$ is a compact Lie group, a discrete group or an abelian locally compact group, we constructed in [5] (see also [6]) an equivariant homology and cohomology theory, defined on the category of all $G$-pairs and $G$-maps, which both satisfy all seven equivariant Eilenberg-Steenrod axioms and which have the given covariant coefficient system $k$ and contravariant coefficient system $m$, respectively, as coefficients. We shall here describe a simpler construction of such equivariant homology and cohomology theories which also is valid for an arbitrary topological group $G$. This simpler construction is described in [6] although main use is still made of the original more complicated construction. We shall also discuss some further properties of these equivariant homology and cohomology theories.

For actions by discrete groups equivariant cohomology and homology theories of this type exist before (see G. Bredon [1], [2] and Th. Bröcker [3]).

I have been informed by A. Wasserman that S. Willson has also observed that my original construction can be modified to give the simpler one described below.

1. Equivariant singular theory. In the following $G$ denotes an arbitrary topological group. Let $R$ be a ring with unit. By an $R$-module we mean a unitary left $R$-module.

Definition 1. A family $\mathscr{F}$ of subgroups of $G$ is called an orbit type family for $G$ if the following is true: if $H \in \mathscr{F}$ and $H^{\prime}$ is conjugate to $H$, then $H^{\prime} \in \mathscr{F}$.

Thus, the family of all closed subgroups and the family of all finite subgroups of $G$ are examples of orbit type families for $G$. A more special example is the following. Let $G=O(n)$ and let $\mathscr{F}$ be the family of all subgroups conjugate to $O(m)$ (standard imbedding) for some $m$, where $0 \leqq m \leqq n$.

DEFINITION 2. Let $\mathscr{F}$ be an orbit type family for $G$. A covariant coefficient system $k$ for $\mathscr{F}$ over the ring $R$ is a covariant functor from the category of

AMS (MOS) subject classifications (1970). Primary 55B99, 55B25, 57E99. 
$G$-spaces of the form $G / H$, where $H \in \mathscr{F}$, and $G$-homotopy classes of $G$-maps to the category of $R$-modules.

A contravariant coefficient system $m$ is defined by the contravariant version of the above definition.

THEOREM 1. Let $G$ be a topological group. Let $\mathscr{F}$ be an orbit type family for $G$ and let $k$ be a covariant coefficient system for $\mathscr{F}$ over the ring $R$. Then there exists an equivariant homology theory $H_{*}^{G}(; k)$ defined on the category of all G-pairs and all G-maps (and with values in the category of $R$-modules) which satisfies all seven equivariant Eilenberg-Steenrod axioms, and which has the given coefficient system $k$ as coefficients.

By the statement that $H_{*}^{G}(; k)$ satisfies the equivariant dimension axiom and has $k$ as coefficients, we mean the following. If $H \in \mathscr{F}$, then

$$
H_{n}^{G}(G / H ; k)=0 \text { for } n \neq 0,
$$

and there exists an isomorphism

$$
\gamma: H_{0}^{G}(G / H ; k) \cong k(G / H)
$$

which commutes with homomorphisms induced by $G$-maps $\alpha: G / H \rightarrow G / K$, where $H, K \in \mathscr{F}$. The meaning of the rest of Theorem 1 is clear. Let us only point out that the equivariant excision axiom is satisfied in the following strong sense. An inclusion of the form

$$
i:(X-U, A-U) \rightarrow(X, A)
$$

where $\bar{U} \subset A^{\circ}$ ( $U$ and $A$ are $G$-subsets of the $G$-space $X$ ) induces isomorphisms

$$
i_{*}: H_{n}^{G}(X-U, A-U ; k) \cong H_{n}^{G}(X, A ; k)
$$

for all $n$.

THEOREM 2. Let $G$ and $\mathscr{F}$ be as above, and let $m$ be a contravariant coefficient system for $\mathscr{F}$ over the ring $R$. Then there exists an equivariant cohomology theory $H_{G}^{*}(; m)$ defined on the category of all G-pairs and all G-maps which satisfies all seven equivariant Eilenberg-Steenrod axioms and has the given coefficient system $m$ as coefficients.

Construction of $H_{n}^{G}(X ; k)$. Let $\Delta_{n}$ be the standard $n$-simplex. First observe that if $h: \Delta_{n} \times G / K \rightarrow \Delta_{n} \times G / K^{\prime}$, where $K, K^{\prime} \in \mathscr{F}$, is a $G$-map which covers id: $\Delta_{n} \rightarrow \Delta_{n}$ then $h$ determines a unique $G$-homotopy class of $G$-maps from $G / K$ to $G / K^{\prime}$, and thus $h$ induces a unique homomorphism $h_{*}: k(G / K) \rightarrow k\left(G / K^{\prime}\right)$. Let $X$ be a $G$-space. An equivariant singular $n$-simplex in $X$ is a $G$-map $T: \Delta_{n} \times G / K \rightarrow X$, where $K$ is some subgroup 
of $G$. We call $K$ for the type of $T$, and denote $t(T)=K$. Define

$$
\hat{C}_{n}^{G}(X ; k)=\sum_{t(T) \in \mathscr{F}} \oplus\left(Z_{T} \otimes k(G / t(T))\right)
$$

where the direct sum is over all equivariant singular $n$-simplexes $T$ in $X$ with $t(T) \in \mathscr{F}$. Here $Z_{T}$ denotes the infinite cyclic group on the generator $T$, and the tensor product is over the integers. The boundary homomorphism $\hat{\partial}: \hat{C}_{n}^{G}(X ; k) \rightarrow \hat{C}_{n-1}^{G}(X ; k)$ is defined in the usual way. Define a relation $\sim$ (it is not symmetric) among the elements of the form $T \otimes a$, $a \in k(G / t(T))$, in $\hat{C}_{n}^{G}(X ; k)$ as follows. Let $T \otimes a \sim T^{\prime} \otimes a^{\prime}$ if there exists a $G$-map $h: \Delta_{n} \times G / t(T) \rightarrow \Delta_{n} \times G / t\left(T^{\prime}\right)$, which covers id: $\Delta_{n} \rightarrow \Delta_{n}$, such that $T=T^{\prime} h$ and $h_{*}(a)=a^{\prime}$. Define $\bar{C}_{n}^{G}(X ; k)$ to be the submodule of $\hat{C}_{n}^{G}(X ; k)$ consisting of all elements of the form $\sum_{i=1}^{s}\left(T_{i} \otimes a_{i}-T_{i}^{\prime} \otimes a_{i}^{\prime}\right)$, where $T_{i} \otimes a_{i} \sim T_{i}^{\prime} \otimes a_{i}^{\prime}$ or $T_{i}^{\prime} \otimes a_{i}^{\prime} \sim T_{i} \otimes a_{i}, i=1, \ldots, s$. Then define $C_{n}^{G}(X ; k)=\hat{C}_{n}^{G}(X ; k) / \bar{C}_{n}^{G}(X ; k)$ and observe that $\hat{\partial}$ induces a boundary homomorphism $\partial: C_{n}^{G}(X ; k) \rightarrow C_{n-1}^{G}(X ; k)$. Finally define

$$
H_{n}^{G}(X ; k)=n \text {th homology of the chain complex }\left\{C_{n}^{G}(X ; k), \partial_{n}\right\} .
$$

The relative groups $H_{n}^{G}(X, A ; k)$ for a $G$-pair $(X, A)$, the boundary homomorphism $\partial: H_{n}^{G}(X, A ; k) \rightarrow H_{n-1}^{G}(A ; k)$, and induced homomorphisms $f_{*}: H_{n}^{G}(X, A ; k) \rightarrow H_{n}^{G}(Y, B ; k)$ by a $G$-map $f:(X, A) \rightarrow(Y, B)$ are then defined in the obvious way. The equivariant homotopy and excision axioms are proved by imitating the proofs of the homotopy and excision axioms for ordinary singular homology given in Eilenberg-Steenrod [4]. The proof of the equivariant dimension axiom is trivial. We call this equivariant homology theory for "equivariant singular homology with coefficients in $k . "$

The "equivariant singular cohomology theory with coefficients in a contravariant coefficient system $m$ " is constructed by a procedure dual to the one described above.

We shall now formulate the functoriality property in the transformation group $G$. Let $G^{\prime}$ be another topological group, $\mathscr{F}^{\prime}$ an orbit type family for $G^{\prime}$, and $k^{\prime}$ a covariant coefficient system for $\mathscr{F}^{\prime}$ over $R$. Assume that $\varphi: G^{\prime} \rightarrow G$ is a continuous homomorphism such that $\varphi\left(H^{\prime}\right) \in \mathscr{F}$ if $H^{\prime} \in \mathscr{F}$. Let $\Phi: k^{\prime} \rightarrow k$ be a natural transformation of covariant coefficient systems with respect to the homomorphism $\varphi: G^{\prime} \rightarrow G$. By this we mean that for each $H^{\prime} \in \mathscr{F}^{\prime}$ we have a homomorphism $\Phi: k^{\prime}\left(G^{\prime} / H^{\prime}\right) \rightarrow k\left(G / \varphi\left(H^{\prime}\right)\right)$, such that if $K^{\prime} \in \mathscr{F}^{\prime}$ and $\alpha: G^{\prime} / H^{\prime} \rightarrow G^{\prime} / K^{\prime}$ is a $G^{\prime}$-map, then $\Phi \alpha_{*}=\varphi(\alpha)_{*} \Phi$. Here $\varphi(\alpha): G / \varphi\left(H^{\prime}\right) \rightarrow G / \varphi\left(K^{\prime}\right)$ is given by $\varphi(\alpha)\left(g \varphi\left(H^{\prime}\right)\right)=g \varphi\left(g_{0}^{\prime}\right) \varphi\left(K^{\prime}\right)$ where $\alpha\left(g^{\prime} H^{\prime}\right)=g^{\prime} g_{0}^{\prime} K^{\prime}$.

If $Y$ is a $G$-space we denote by $Y^{\prime}$ the same topological space considered as a $G^{\prime}$-space through $\varphi$; that is, $G^{\prime}$ acts on $Y^{\prime}$ by $g^{\prime} y=\varphi\left(g^{\prime}\right) y, y \in Y^{\prime}=Y$, $g^{\prime} \in G^{\prime}$. 
Proposition 3. Let the notation be as above and let $(Y, B)$ be a G-pair. Then we have induced homomorphisms

$$
(\varphi, \Phi)_{*}: H_{n}^{G^{\prime}}\left(Y^{\prime}, B^{\prime} ; k^{\prime}\right) \rightarrow H_{n}^{G}(Y, B ; k)
$$

for all $n$, which commute with the boundary homomorphism and with homomorphisms induced by G-maps.

Next we consider transfer homomorphisms. Let $A \subset G$ be a closed subgroup of $G$, such that $A \backslash G$ is a finite set. We say that a $G$-map $\beta: G / H \rightarrow G / K$ is of type " $A$ " if we have $\beta(e H)=a_{0} K$, where $a_{0} \in A$. If $\beta$ is of type " $A$ " we can define an $A$-map $\beta^{!}: A / A \cap H \rightarrow A / A \cap K$ by the condition $\beta^{!}(e(A \cap H))=a_{0}(A \cap K)$. Let $\mathscr{F}^{\prime}$ be an orbit type family for $A$, such that if $H \in \mathscr{F}$ then $H \cap A \in \mathscr{F}^{\prime}$, and let $k^{\prime}$ be a covariant coefficient system for $\mathscr{F}^{\prime}$ over $R$. Let $\Phi^{!}: k \rightarrow k^{\prime}$ be a natural transformation of transfer type with respect to the inclusion $A \hookrightarrow G$. By this we mean that for every $H \in \mathscr{F}$ we have a homomorphism $\Phi^{!}: k(G / H) \rightarrow$ $k^{\prime}(A / A \cap H)$ such that if $K \in \mathscr{F}$ and $\beta: G / H \rightarrow G / K$ is a $G$-map of type " $A$ " then $\Phi^{!} \beta_{*}=\left(\beta^{!}\right)_{*} \Phi^{!}$. If $Y$ is a $G$-space we denote by $Y^{\prime}$ the $A$-space obtained by restricting the action to the subgroup $A$.

Proposition 4. Let the notation be as above and let $(Y, B)$ be a G-pair. Then we have transfer homomorphisms

$$
\tau^{!}: H_{n}^{G}(Y, B ; k) \rightarrow H_{n}^{A}\left(Y^{\prime}, B^{\prime} ; k^{\prime}\right)
$$

for all $n$, which commute with the boundary homomorphism and with homomorphisms induced by G-maps.

Assume that $A \backslash G$ consists of $s$ elements and that the orbit type family $\mathscr{F}$ for $G$ is such that if $H \in \mathscr{F}$ then also $A \cap H \in \mathscr{F}$. Also assume that for each $H \in \mathscr{F}$ the composite homomorphism

$$
k(G / H) \stackrel{\Phi^{!}}{\rightarrow} k^{\prime}(A / A \cap H) \stackrel{\Phi}{\rightarrow} k(G / A \cap H) \stackrel{p_{*}}{\rightarrow} k(G / H)
$$

equals the identity.

Proposition 5. Let the notation be as above. Then the composite

$$
H_{n}^{G}(Y ; k) \stackrel{\tau^{!}}{\rightarrow} H_{n}^{A}\left(Y^{\prime} ; k^{\prime}\right) \stackrel{(i, \Phi)_{*}}{\longrightarrow} H_{n}^{G}(Y ; k)
$$

equals multiplication by $s$.

The corresponding cohomology versions of Propositions 3-5 are also valid.

Let $k$ and $m$ be a covariant and a contravariant coefficient system, respectively, over a commutative ring $R$. A pairing $\omega$ of $k$ and $m$ consists 
of the following. For each $H \in \mathscr{F}$ we have a homomorphism of $R$-modules

$$
\omega: m(G / H) \otimes_{R} k(G / H) \rightarrow R
$$

such that if $K \in \mathscr{F}$ and $\alpha: G / H \rightarrow G / K$ is a $G$-map then

$$
\omega\left(a \otimes_{R} \alpha_{*}(b)\right)=\omega\left(\alpha_{*}(a) \otimes_{R} b\right), \quad a \in m(G / K), b \in k(G / H) .
$$

Such a pairing $\omega$ of $m$ and $k$ gives rise to a pairing

$$
\langle,\rangle: H_{G}^{n}(X ; m) \otimes_{R} H_{n}^{G}(X ; k) \rightarrow R
$$

which we call the Kronecker index. The Kronecker index gives rise to the homomorphism

$$
v: H_{G}^{n}(X ; m) \rightarrow \operatorname{Hom}_{R}\left(H_{n}^{G}(X ; k), R\right)
$$

defined by $v(\eta)(\xi)=\langle\eta, \xi\rangle$, where $\eta \in H_{G}^{n}(X ; m)$ and $\xi \in H_{n}^{G}(X ; \mathrm{k})$.

We say that a contravariant coefficient system $m$ is a commutative ring coefficient system if $m(G / H)$ is a commutative ring for each $H \in \mathscr{F}$ and all induced homomorphisms are ring homomorphisms, and moreover $m(G / G)=R$ and the $R$-module structure on $m(G / H)$ is the same as the one induced by the ring homomorphism $p^{*}: R=m(G / G) \rightarrow m(G / H)$. If $m$ is a commutative ring coefficient system, we have cup-products

$$
\bigcup: H^{n}(X ; m) \otimes_{R} H^{p}(X ; m) \rightarrow H^{n+p}(X ; m) .
$$

\section{REFERENCES}

1. G. Bredon, Equivariant cohomology theories, Bull. Amer. Math. Soc. 73 (1967), 266-268. MR 34 \#6762.

2. Verlag, Berlin and New York, 1967. MR 35 \# 4914.

3. Th. Bröcker, Singuläre Definition der Äquivarianten Bredon Homologie, Manuscripta Math. 5 (1971), 91-102.

4. S. Eilenberg and N. E. Steenrod, Foundations of algebraic topology, Princeton Univ. Press, Princeton, N.J., 1952. MR 14, 398.

5. S. Illman, Equivariant singular homology and cohomology for actions of compact Lie groups, Proc. Conference on Transformation Groups (University of Massachusetts, Amherst, 1971), Lecture Notes in Math., Springer-Verlag, volume 298, Berlin and New York, 1972.

6. - Equivariant algebraic topology, Thesis, Princeton University, Princeton, N.J., 1972.

Department of Mathematics, Princeton University, Princeton, New Jersey 08540

Current address: Department of Mathematics, Helsinki University, Hallituskatu, Helsinki, Finland 